\title{
ADDITION OF MILK ENHANCED THE TOTAL PHENOLIC CONTENT AND ANTIOXIDANT ACTIVITY OF Camellia sinensis (GREEN TEA) BEVERAGES
}

\author{
KEE XIAO HUI and SITI RAIHANAH SHAFIE* \\ Department of Nutrition, Faculty of Medicine and Health Sciences, \\ Universiti Putra Malaysia, 43400 UPM, Serdang, Selangor, Malaysia \\ *E-mail: sitiraihanah@upm.edu.my
}

Accepted 4 October 2021, Published online 31 December 2021

\begin{abstract}
The study was aimed to determine the effects of adding milk, sugar and sweeteners to the total phenolic content and antioxidant activity of Camellia Sinensis (green tea) powder. Six green tea samples were brewed in cold water and whisked: Green tea powder (G), green tea powder with sugar (GS), green tea powder with aspartame (GA), green tea powder with milk (GM), green tea powder with milk and sugar (GMS) and green tea powder with milk and aspartame (GMA). The total phenolic content of green tea powder samples was evaluated by using Folin-Ciocalteu assay while $\alpha, \alpha$-diphenyl-2picrylhydrazyl (DPPH) and ferric reducing antioxidant power (FRAP) assays were used to measure the antioxidant activity. Results showed that milk addition significantly enhanced by $59.84 \%$ of total phenolic content and also increased $26.3 \%$ (DPPH) and $42.35 \%$ (FRAP) of antioxidant activity of green tea powder, as compared to green tea samples without milk $(p<0.05)$. Strong and positive correlations were found between the total phenolic content and antioxidant activity of green tea powder by using DPPH $(r=0.990)$ and FRAP assays $(r=0.997)$. In conclusion, it might be beneficial when milk is added to green tea as it could help to increase its antioxidant properties.
\end{abstract}

Key word: Antioxidant, artificial sweetener, green tea, milk, sugar

\section{INTRODUCTION}

Drinking tea (Camellia sinensis) and tea culture is one of the cultural heritages dated 5000 years ago (Kumarihami \& Song, 2018) and is recognized as a popular beverage in the world (Chu \& Pang, 2018; Prasanth et al., 2019; Musial et al., 2020; Yan et al., 2020). Green tea is classified as a functional beverage and it is widely consumed by people worldwide, due to its health benefits (Hayat et al., 2015; Prasanth et al., 2019). Green tea consists of a wide array of polyphenols, and flavan-3-ols (catechins) made up of around $90 \%$ of its polyphenol constituents (Lee et al., 2014). They play an important role in human health as powerful free radical scavengers and enzyme activity regulators for different types of oxidases (Yan et al., 2020). Previous studies have shown that green tea consumption brings healthpromoting effects, including anti-atherosclerosis, anti-mutagenic, anti-inflammatory, anti-diabetic, anti-

\footnotetext{
* To whom correspondence should be addressed.
}

obesity, anti-viral, and neuroprotective effects (Li \& Zhu, 2015; Chu et al., 2017; Reygaert, 2018).

Matcha is a special powdered form of green tea of the "Tencha" type, which originated from the traditional tea culture in Japan (Horie et al., 2017; Sakurai et al., 2020). The cultivation methods and process for "Tencha" tea production are different from the normal green tea, although they are derived from the same tea plant (Camellia Sinensis) (Sakurai et al., 2020). "Tencha" is cultivated under $60-90 \%$ shade from direct sunlight for about 7-10 or 21 days before harvest, depending on the demographic factor, tea farmer's experience as well as the age of the tea tree (Ku et al., 2010; Sakurai et al., 2020). After that, the stems and the stems and veins are removed from the tea leaves, and steamed soon after being harvested (Sakurai et al., 2020). The drying process is carried out subsequently by using the "Tencharo" without undergoing the rolling process (Horie et al., 2017). The dried tea leaves are then grinded into powdered form by using the exclusive stone mills, known as "matcha". In other words, matcha is 
consumed in whole tea leaves, as compared to other kinds of tea (Koláčková et al., 2020). Therefore, matcha contains higher nutrient as well as polyphenol content than green tea leaves, due to its unique cultivation and processing method.

Adding secondary ingredients such as milk and sugar into tea is a common practice to reduce the astringency of the tea (Bhagat et al., 2019). Nowadays, drinking sweetened milk tea had gained high popularity across the current market. These are varieties of milk tea available worldwide, such as bubble milk tea, Hong Kong milk tea, Thai milk, Masala Chai, and British milk tea are commonly consumed by people, depending on the cultures and regions. Hence, it is important to know whether the milk and sugar addition will affect the antioxidant capacity of tea or not. However, it remained controversial as the previous studies showed the different results, whether the milk addition will decrease the total antioxidant of tea (Hertog et al., 1997; Tewari et al., 2000; Langley-Evans, 2000; Lorenz et al., 2007; Kartsova \& Alekseeva, 2008; Muthuiah et al., 2009; Ryan \& Petit, 2010; Ryan \& Sutherland, 2011; Egert et al., 2013; Ye et al., 2013; Korir et al., 2014; Wipatanawin et al., 2015; Imran et al., 2017); no significant effect on the tea antioxidant capacity (Van Het Hof et al., 1998; Leenen et al., 2000; Hollman et al., 2001; Richelle et al., 2001; Reddy et al., 2005; Kyle et al., 2007; Rashidinejad et al., 2016a; Bartoszek et al., 2018); or enhanced antioxidant activity of tea (Mirzaei \& Mirzaei, 2013; Xie et al., 2013; Otemuyiwa et al., 2017;), based on the different experimental designs and type of teas used. Similarly, mixed finding found in the investigation on the impact of adding sugar to the total antioxidant activity of tea, whether it will decrease the antioxidant (Muthuiah et al., 2009; Korir et al., 2014); no significant effect; (Shalaby et al., 2016; Otemuyiwa et al., 2017), or dual effects (Sharma et al., 2008; Wipatanawin et al., 2015; Shalaby et al., 2016; Otemuyiwa et al., 2017), depends on the different assay used. There are also the increasing trends of applying artificial sweetener in the food and beverage industry in order to cut off the excessive calories and sugar intake. Therefore, it is also urged to know that whether the artificial sweetener addition will influence the total antioxidant capacity of tea or not. There were only a few studies investigated on the impact on this aspect (Ryan \& Sutherland, 2011; Korir et al., 2014; Shalaby et al., 2016; Wipatanawin et al., 2015).

In short, the purpose of this study is to investigate the effects of adding milk, sugar, and artificial sweetener to the antioxidant capacities and total antioxidant content of green tea (Camellia sinensis).

\section{MATERIALS AND METHODS}

\section{Sample preparation}

Green tea powder (matcha) was purchased from one local store (Niko Neko Matcha) in Kuala Lumpur, Malaysia. Tea samples were prepared based on the ratio of the drink available from the recipe given by the supplier. Potable water was used to replace the milk to prepare the tea beverages without the addition of milk. The sample preparation was summarized in Figure 1.

Firstly, six types of tea brews were prepared and labeled as followed: green tea powder $(\mathrm{G})$, green tea powder with sugar (GS), green tea powder with aspartame (GA), green tea powder with milk (GM), green tea powder with milk and sugar (GMS) and green tea powder with milk and aspartame (GMA). Coldwater was used to prepare the beverages as suggested by the manufacturer. A milk frother was used to whisk the beverages to ensure that the mixture is evenly blended and no clumps were left in the mixture. The fresh samples were ready to use for assessing the total phenolic content and the antioxidant activity.

\section{Chemicals and standards}

DPPH (2,2-diphenyl-1-picrylhydrazyl radicals), Trolox were purchased from Sigma-Aldrich, Malaysia. Folin-Ciocalteu's reagent, sodium carbonate, ethyl acetate, acetic acid, and perhydrol stabilized 30\% $\mathrm{H}_{2} \mathrm{O}_{2}$ were from Sigma-Aldrich, Malaysia. Ferric tripyridyltriazine ( $\left.\mathrm{Fe}^{3+-} \mathrm{TPTZ}\right)$, gallic acid was purchased from Sigma-Aldrich, Malaysia.

\section{Total phenolic content}

The total phenolic content of each sample was determined by using the Folin-Ciocalteu method (Singleton \& Rossi, 1965) with some modifications done by Ramírez-Aristizabal et al. (2017). Briefly, 160 $\mu \mathrm{L}$ of the $5 \%$ sample was pipetted into the screwcap test tube. After that, $1420 \mu \mathrm{L}$ of $10 \%$ of FolinCiocalteu reagent and $1420 \mu \mathrm{L}$ of $7.5 \%$ sodium carbonate were added, vortexed, and mixed well together with the sample. Then, the sample was incubated in the dark for $2 \mathrm{~h}$ at the ambient temperature. Absorbance was determined at $758 \mathrm{~nm}$ by using a UV-Visible spectrophotometer after $2 \mathrm{~h}$. The total phenolic content of the sample was assessed by using a gallic acid standard calibration curve $(0$ to $240 \mathrm{mg} / \mathrm{L})$. The results are expressed as mg gallic acid equivalent per litre per sample (mg GAE/L per sample). 


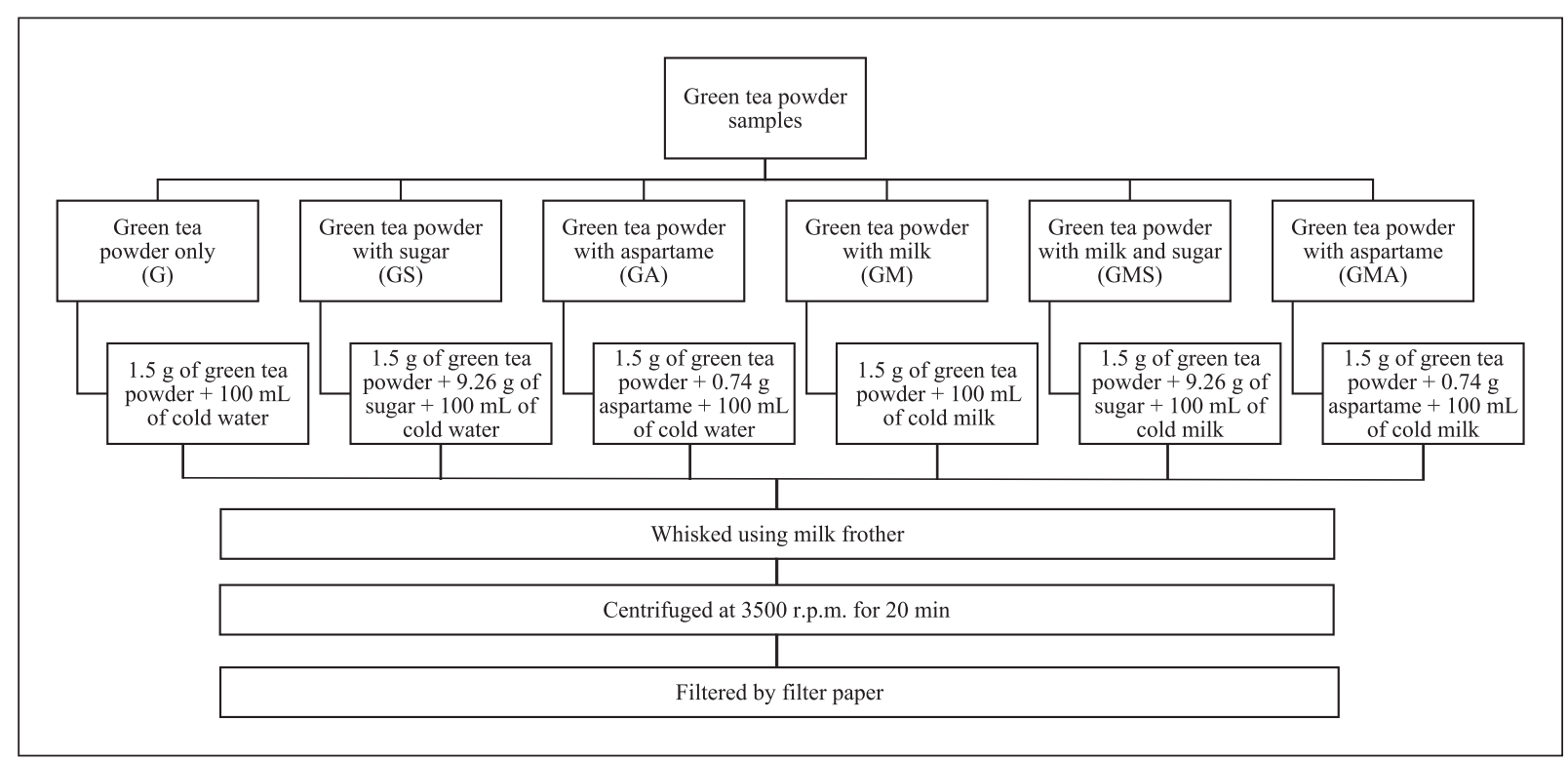

Fig. 1. Steps of preparing samples.

\section{Total Antioxidant Activity}

$\alpha, \alpha$-Diphenyl- $\beta$-Picrylhydrazyl (DPPH) assay

$\alpha, \alpha$-Diphenyl- $\beta$-Picrylhydrazyl (DPPH) assay was used for determining the effects of adding milk, sugar, and artificial sweetener to the total antioxidant activity of green tea. DPPH assay by Singleton and Rossi (1965) with some modifications described by Ghasemzadeh-mohammadi et al. (2017).

Firstly, $0.1 \mathrm{~mL}$ of sample and $3.0 \mathrm{~mL}$ of ethanolic DPPH solution were introduced, mixed, and vortexed in the test tube. Then, the sample was incubated in the dark for $30 \mathrm{~min}$ at room temperature. After $30 \mathrm{~min}$, the clear solution had been taken out and measured the absorbance at $517 \mathrm{~nm}$ by using a UV-Visible spectrophotometer. Each sample was measured in triplicate. At the same time, $0.1 \mathrm{~mL}$ of distilled water and $3.0 \mathrm{~mL}$ of ethanolic DPPH solution were prepared as a control.

The percentage of scavenging activity or antioxidant activity (AA) percentage was calculated by using the following formula:

Free radical scavenging ability $(\%)=\frac{A b s_{\text {control }}-A b s_{\text {blank }}}{A b s_{\text {control }}} \times 100$

Ferric Iron Reducing Antioxidant Power (FRAP)

FRAP assay (Benzie \& Strain, 1996) was applied in this study to determine the total antioxidant activity of the samples with some modification as described by Xiao et al. (2020).

Firstly, $0.125 \mathrm{~mL}$ of $5 \%$ sample was mixed thoroughly with $4.5 \mathrm{~mL}$ of the FRAP reagent, vortexed, and then incubated in the dark for $15 \mathrm{~min}$ at $37^{\circ} \mathrm{C}$. The ferric tripyridyltriazine $\left(\mathrm{Fe}^{3+}-\mathrm{TPTZ}\right)$ complex will reduce into ferrous tripyridyltriazine ( $\left.\mathrm{Fe}^{2+}-\mathrm{TPTZ}\right)$ and intense blue color could be observed when there is a presence of antioxidant in the sample.

At the same time, a Trolox standard calibration curve in the range of 31.25 to $4000 \mu \mathrm{M}$ were prepared with linear equation $\mathrm{y}=0.0005 \mathrm{x}+0.0883$, $\left(R^{2}=0.9734\right)$. The absorbance at $593 \mathrm{~nm}$ was recorded after the sample was tested by using a UV-Vis spectrophotometer. Each sample was measured in triplicate. The results are expressed mean and standard deviation $(\mu \mathrm{M})$ of Trolox equivalent (TE) per liter (L) of the sample.

\section{Statistical analysis}

IBM SPSS Statistics 25 was applied to perform all the statistical analyses. A one-way ANOVA with post-hoc Tukey HSD Test was used in this study to determine the differences of total phenolic content as well as a total antioxidant activity among all the samples. Pearson's correlation analysis was also carried out to determine the relationship between the total phenolic content as well as the total antioxidant activity among the samples. Statistical comparisons were considered significant at $p<0.05$.

\section{RESULTS AND DISCUSSION}

\section{Total phenolic content}

Table 1 shows the total phenolic content of different green tea powder samples.

Based on the results obtained, the descending order of total phenolic content among different green tea powder samples was GMA (2916.67 $\pm 42.76 \mathrm{mg}$ 
Table 1. Total phenolic content of different green tea powder samples

\begin{tabular}{lc}
\hline Green tea powder samples $(n=6)$ & $\begin{array}{c}\text { Total Phenolic Content } \\
\text { (mg GAE equivalent /L) }\end{array}$ \\
\hline Green tea powder only (G) & $1150.00 \pm 141.01^{\mathrm{a}}$ \\
Green tea powder with sugar (GS) & $1194.44 \pm 31.55^{\mathrm{a}}$ \\
Green tea powder with aspartame (GA) & $1200.00 \pm 41.94^{\mathrm{a}}$ \\
Green tea powder with milk (GM) & $2863.89 \pm 16.67^{\mathrm{b}}$ \\
Green tea powder with milk and sugar (GMS) & $2888.89 \pm 36.32^{\mathrm{b}}$ \\
Green tea powder with milk and aspartame (GMA) & $2916.67 \pm 42.76^{\mathrm{b}}$ \\
\hline
\end{tabular}

Values are expressed as mean \pm standard deviation $(n=6)$. Means with different letters are significantly different at $p<0.05$.

GAE/L $)>$ GMS $(2888.89 \pm 36.32 \mathrm{mg} \mathrm{GAE} / \mathrm{L})>\mathrm{GM}$ $(2863.89 \pm 16.67 \mathrm{mg} \mathrm{GAE} / \mathrm{L})>\mathrm{GA}(1200.00 \pm 41.94$ $\mathrm{mg} \mathrm{GAE} / \mathrm{L})>\mathrm{GS}(1194.44 \pm 31.55 \mathrm{mg} \mathrm{GAE} / \mathrm{L})>\mathrm{G}$ $(1150.00 \pm 141.01 \mathrm{mg}$ GAE/L). The result shows that milk addition had significantly increased the total phenolic content in green tea powder (GM) by $59.84 \%$ as compared to green tea powder only $(G)$. There was a significant difference between the green tea powder samples with added milk (GM, GMS, GMA) and those green tea powder samples that did not add milk (G, GS, GA) $(p<0.001)$.

Briefly, there was a significantly higher total phenolic content in the matcha infusion with milk addition (GM, GMS, \& GMA), compared to the green tea powder without adding milk (G, GS, and GA). This was in line with the result obtained by Otemuyiwa et al. (2017), who reported that there was a significant enhancement after the milk was poured into both black and green tea samples. This was plausible that the overestimation of the total phenolic content of green tea powder itself by Folin-Ciocalteu reagent. Some studies found that the Folin-Ciocalteu reagent may overestimate the antioxidant substances from food samples (Everette et al., 2014; Rashidinejad et al., 2016b; Cutrim \& Cortez, 2018). As FolinCiocalteu reagent was formerly used to determine tryptophan (an aromatic amino acid which is a nonphenolic group), and hence the protein content might contribute a higher phenolic content from the food samples by reducing the Folin-Ciocalteu reagent into blue-green complex (Everette et al., 2010). This indicated that the Folin Ciocalteu reagent might not only detect the total phenolic content from green tea powder but also the protein content contributed by the milk. However, this result was contradicted with the majority of the earlier studies, which stated that the total phenolic content in the tea-milk infusions was reduced significantly, due to the formation of milk protein-polyphenols complex (Sharma et al., 2008; Imran et al., 2017). There was no significant changes of total phenolic content between the black tea-milk infusion and the black tea-only infusion found in Bartoszek et al. (2018)'s study.
Furthermore, G, GS, and GA shared similar results and they had no significant differences from each other. This indicated that there was no significant impact on the total phenolic content when adding sugar and aspartame into green tea powder. This result was similar to the previous studies by Sharma et al. (2008), which reported that the sugaradded black tea stabilized the total antioxidant activity, although the total phenolic content of the black tea with sugar infusion decreased marginally. A contradicted result found that the total phenolic content of green tea increased along with the sugar amount added (4-12 $\mathrm{mg}$ of sugar/ $\mathrm{mL}$ of tea), but reduced when more sugar was added (Otemuyiwa et al., 2017). Besides that, a study showed that there was a significant enhancement of total phenolic content of sugared-black tea but the opposite effect was found in sugared-green tea (Shalaby et al., 2016). There was no significant difference from each other for GM, GMS, and GMA. This indicates that there is no impact on the total phenolic content with adding sugar and aspartame into the green tea powder with milk.

\section{Total antioxidant activity}

\section{1,1-diphenyl-2-picryl hydroxyl radical (DPPH)}

Table 2 summarizes the percentage of the free radical scavenging ability of different green tea powder samples.

DPPH radical scavenging activity among different green tea samples were arranged as followed: GMS $(93.7 \pm 0.57 \%)>$ GMA $(93.4 \pm 0.38 \%)$ $>\mathrm{GM}(92.8 \pm 0.71 \%)>\mathrm{GS}(66.8 \pm 0.78 \%)>\mathrm{GA}(66.6$ $\pm 1.21 \%)>\mathrm{G}(66.5 \pm 1.17 \%)$. It revealed that there was a remarkable contrast between the milk-addition to green tea powder samples (GM, GMS, GMA) and the green tea powder samples with no added milk $(\mathrm{G}$, GS, GA), $F(5,12)=869.202, p<0.001$.

The total antioxidant activity of the green tea powder had significantly increased $(p<0.001)$ 26.3\% after adding milk, compared to those green tea powder samples without adding milk. This finding 
Table 2. Free radical scavenging ability of different green tea powder samples

\begin{tabular}{lc}
\hline Green tea powder samples $(n=6)$ & Free radical scavenging ability $(\%)$ \\
\hline Green tea powder only (G) & $66.5 \pm 1.17^{\mathrm{a}}$ \\
Green tea powder with sugar (GS) & $66.8 \pm 0.78^{\mathrm{a}}$ \\
Green tea powder with aspartame (GA) & $66.6 \pm 1.21^{\mathrm{a}}$ \\
Green tea powder with milk (GM) & $92.8 \pm 0.71^{\mathrm{b}}$ \\
Green tea powder with milk and sugar (GMS) & $93.7 \pm 0.57^{\mathrm{b}}$ \\
Green tea powder with milk and aspartame (GMA) & $93.4 \pm 0.38^{\mathrm{b}}$ \\
\hline
\end{tabular}

Values are expressed as mean \pm standard deviation $(n=6)$. Means with different letters are significantly different at $p<0.05$.

was paralleled to the earlier studies that both black and green tea exhibited enhanced free radical scavenging ability by using the DPPH method (Mirzaei \& Mirzaei, 2013; Otemuyiwa et al., 2017). The authors suggested that the enhancing effect on the antioxidant activity of tea by adding milk, may not be solely due to the phenolic-protein interaction, but also protein-protein interaction, particularly the reduced collision length of protein molecules was observed when the amount of milk increased (Otemuyiwa et al., 2017). There was also possible that the cold brewing temperature leads to the antioxidant activity enhancement in tea. Xie et al. (2013) observed that hot preparation of tea + milk samples had poor catechin recoveries, as compared to the cold green tea extraction preparation with milk, due to high temperature is favorable for catechin to form complexes with milk protein. More haze will be formed when the milk was added into the hot tea solution, and thus more hydrophobic sites, which are usually concentrated in the interior of hydrophilic proteins will be exposed to the catechins (Siebert et al., 1996). However, most of the studies reported the opposite findings, where milk addition will significantly reduce the ability to reduce the free radical level of tea with DPPH assay (Korir et al., 2014; Imran et al., 2017; Sharma et al., 2008; Muthuiah et al., 2009) due to the hydrophobic interaction between the milk protein and polyphenols molecule in tea. Apart from that, no significant impact showed on the ability to quench the free radicals in tea after milk was added (Bartoszek et al., 2018).

No significant difference of the total antioxidant activity within green tea powder samples without milk (G, GS, GA) as well as within green tea powder samples with milk (GM, GMS, GMA) was found in this study. This reflected that sugar or sweetener's addition will not influence the total antioxidant activity of the green tea powder sample. This result was in agreement with the previous studies conducted by Korir et al. (2014) and Shalaby et al. (2016), who reported that there was no statistical difference between the tea with artificial sweeteners (stevia and aspartame) and tea alone. Besides, from the perspective of sugared tea, the result of the total antioxidant activity of black tea remained unchanged even after adding sugar was supported by Bartoszek et al. (2018) and Sharma et al. (2008)'s findings. There were also a few studies that gave a different point of view regarding the finding, where the addition of sugar or sweeteners will have different impacts on the total antioxidant activity of tea. For instance, a notable decline in total antioxidant activity was found in green tea with sugar infusion when the sugar concentration increases (Shalaby et al., 2016). Their findings were in agreement with the previous studies that added sugar into the black tea infusion (Muthuiah et al., 2009; Korir et al., 2014). It is possible the sugar amount added into the tea samples in this present study might not be as much as the sugar added in the previous study and thus no significant impact was found. However, the mechanism of the reaction between the sugar molecule and the tea polyphenols has not yet been studied well.

\section{Ferric Iron Reducing Antioxidant Power (FRAP)}

Table 3 shows the FRAP values of different green tea powder samples with different ingredients added to them.

The FRAP values among different green tea powder samples was assessed and was ranked as followed: GMA $(16494.67 \pm 380.18 \mu \mathrm{m} \mathrm{TE} / \mathrm{L})>\mathrm{GMS}$ $(16348.00 \pm 652.25 \mu \mathrm{m} \mathrm{TE} / \mathrm{L})>\mathrm{GM}(15428.00 \pm 794.31$ $\mu \mathrm{m} \mathrm{TE} / \mathrm{L})>\mathrm{GS}(8948.00 \pm 100.66 \mu \mathrm{m} \mathrm{TE} / \mathrm{L})>\mathrm{GA}$ $(8908.00 \pm 161.66$ ìm TE/L $)>\mathrm{G}(8894.67 \pm 140.48 \mu \mathrm{m}$ $\mathrm{TE} / \mathrm{L})$. There was a significant difference between total antioxidant capacity in the green tea powder with milk samples (GM, GMS, GMA) and green tea powder samples without milk addition (G, GS, GA), $p<0.001$. Therefore, there is no significant difference between the FRAP values of GMA, GMS, and GM, even though GMA had the highest FRAP value.

A Tukey-post hoc test was conducted that the milk-fortified green tea powder samples were statistically higher total antioxidant activity than the green tea powder samples with no added milk. 
$42.35 \%$ higher FRAP value was found in GM as compared to G. This indicated that milk addition to green tea powder was able to improve the total antioxidant activity of tea. To date, no study reported that a significant enhancement of total antioxidant activity presents in milky tea by using FRAP test. However, some in vivo studies revealed that no significant changes in plasma antioxidant activity of the subjects after ingesting milk tea (Leenen et al., 2000; Reddy et al., 2005; Kyle et al., 2007). In contrast, there were a few studies reported that a significant decline in plasma antioxidant activity of subjects when the milk was added into tea (LangleyEvans, 2000; Ryan \& Petit, 2010; Ryan \& Sutherland, 2011). It was remarkable that the changes in total antioxidant activity of milk tea may determine by the milk fat percentage. Ryan and Petit (2010)'s study showed that the antioxidant activity of black tea had reduced significantly the most in tea with skimmed milk, followed by semi-skimmed milk and whole milk. From most of the previous studies, the decline in total antioxidant activity of milk tea was observed when the skimmed/semi-skimmed milk was added instead of whole milk (Van Het Hof et al., 1998; Langley-Evans, 2000; Lorenz et al., 2007; Ryan \& Sutherland, 2011; Egert et al., 2013; Xie et al., 2013) while no significant changes (Leenen et al., 2000; Bartoszek et al., 2018), or even enhance in total antioxidant activity in tea (Mirzaei \& Mirzaei, 2013) were reported when the whole milk was used. It might be because milk contains numerous fat-soluble antioxidants such as vitamin $\mathrm{E}$ and vitamin $\mathrm{A}$ (Rashidinejad et al., 2016b), and thus, skimming off the milk fat might be one of the important factors to reduce its antioxidant activity (Langley-Evans, 2000; Ryan \& Petit, 2010).

Besides, no significant difference between the green tea with sugar or sweetener addition and the green tea powder samples without sugar or sweetener addition. This indicated that there was no significant impact on adding the sweeteners (sugar or aspartame) to the total antioxidant capacity of the green tea powder samples. This finding was supported by a similar study conducted by Ryan and Sutherland (2011), who added the sugar and Canderel sweetener (a kind of artificial sweetener) into black tea, and no significant impact on total antioxidant activity was found. Similarly, Wipatanawin et al. (2015) found that no significant difference between the sugared oolong tea and non-sugar oolong tea by using 2,2-azinobis-(3-ethylbenzothiazoline-6sulfonate) (ABTS+) and ferrous ion-chelating (FIC) and cellular antioxidant activity (CAA) assays. Nevertheless, Wipatanawin et al. (2015) pointed out that the sweetener addition will have a diverse impact on the antioxidant activity of the tea, depending on the different types of tests used to assess the total antioxidant activity. The total antioxidant activity of the oolong tea was reduced when the sweetener was added when using the CAA method but enhanced while using the ABTS+ and FIC assays. The authors proposed that the increased antioxidant activity of the oolong tea with the sweetener addition (contained $98.40 \%$ erythritol \& sucralose $1.56 \%$ ), was due to the powerful free radical scavenging properties possessed by the erythritol. In contrast, in the current study, aspartame was used instead of erythritol. Since aspartame is made up of a methyl ester of the aspartate and phenylalanine dipeptide (Chattopadhyay et al., 2014; Adaramoye \& Akanni, 2016), with no antioxidant properties possessed by them, and thus, the antioxidant capacity of green tea powder was not affected by the addition of aspartame.

\section{Correlation between antioxidant content and antioxidant activity}

A Pearson correlation coefficient test had been carried out to investigate the correlation between the total phenolic content and the antioxidant activity of the green tea powder samples as shown in Table 4. A strong positive correlation was found $(r=0.997$, $p<0.01)$ between total phenolic content and DPPH radical scavenging activity, which was showed that it is statistically significant, respectively. Similarly, there is also strong positive relationship between total phenolic content and FRAP value $(r=0.990$, $p<0.01)$. Both results concluded that the antioxidant activity will increase along with the total phenolic content.

\section{CONCLUSION}

In conclusion, this study demonstrated the impact on the antioxidant properties of green tea powder when adding the secondary ingredients (milk, sugar, and artificial sweetener). The results showed that milk addition enhanced the total phenolic content and total antioxidant activity in green tea powder while the addition of sugar and aspartame did not influence the antioxidant content as well as its activity in green tea. Therefore, the enhancement in antioxidant properties of green tea powder might bring benefits to the consumers who like to drink milk tea beverages. However, the amount of the sugar or aspartame should be added rationally while preparing the tea beverages to avoid extra calories intake as well as unknown adverse effects, i.e. reducing antioxidant properties of tea. It also showed that the total phenolic content of green tea powder has a strong and positive correlation with its free radical scavenging activity by using the DPPH test. This indicated that a higher total phenolic content in green tea powder exhibited higher total antioxidant activity. 
Table 3. FRAP values of green tea powder samples added with different types of ingredients $(n=6)$

\begin{tabular}{lc}
\hline Green tea samples $(n=6)$ & FRAP $(\mu M$ TE/L) \\
\hline Green tea powder only (G) & $8894.67 \pm 140.48^{\mathrm{a}}$ \\
Green tea powder with sugar (GS) & $8948.00 \pm 100.66^{\mathrm{a}}$ \\
Green tea powder with aspartame (GA) & $8908.00 \pm 161.66^{\mathrm{a}}$ \\
Green tea powder with milk (GM) & $15428.00 \pm 794.31^{\mathrm{b}}$ \\
Green tea powder with milk and sugar (GMS) & $16348.00 \pm 652.25^{\mathrm{b}}$ \\
Green tea powder with milk and aspartame (GMA) & $16494.67 \pm 380.18^{\mathrm{b}}$ \\
\hline
\end{tabular}

Values are expressed as mean \pm standard deviation $(n=6)$. Means with different letters are significantly different at $p<0.05$.

\section{Appendices}

Appendix A: Gallic acid standard curve for Folin-Ciocalteu assay.

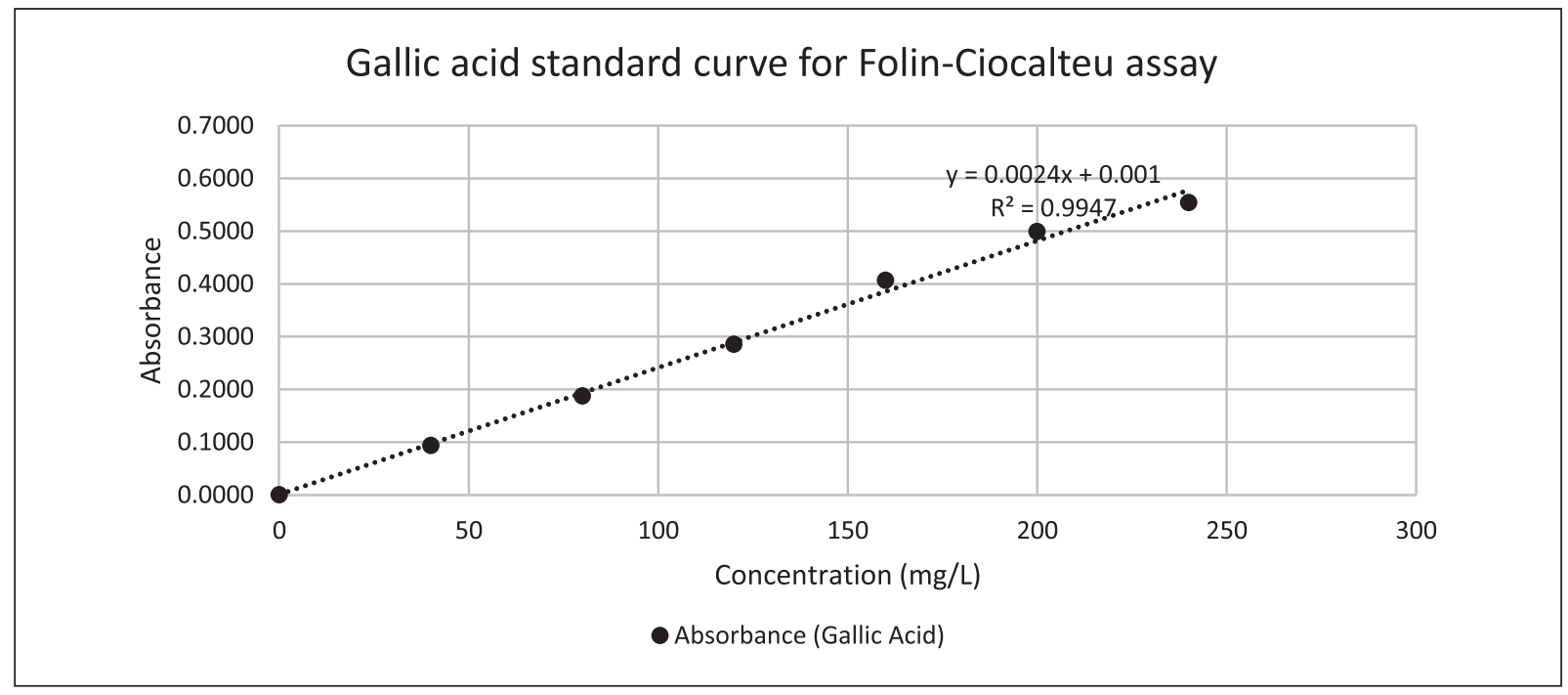

Appendix B: Trolox standard curve for FRAP assay.

\section{Trolox standard curve for Ferric Reducing Ability Power (FRAP) assay}

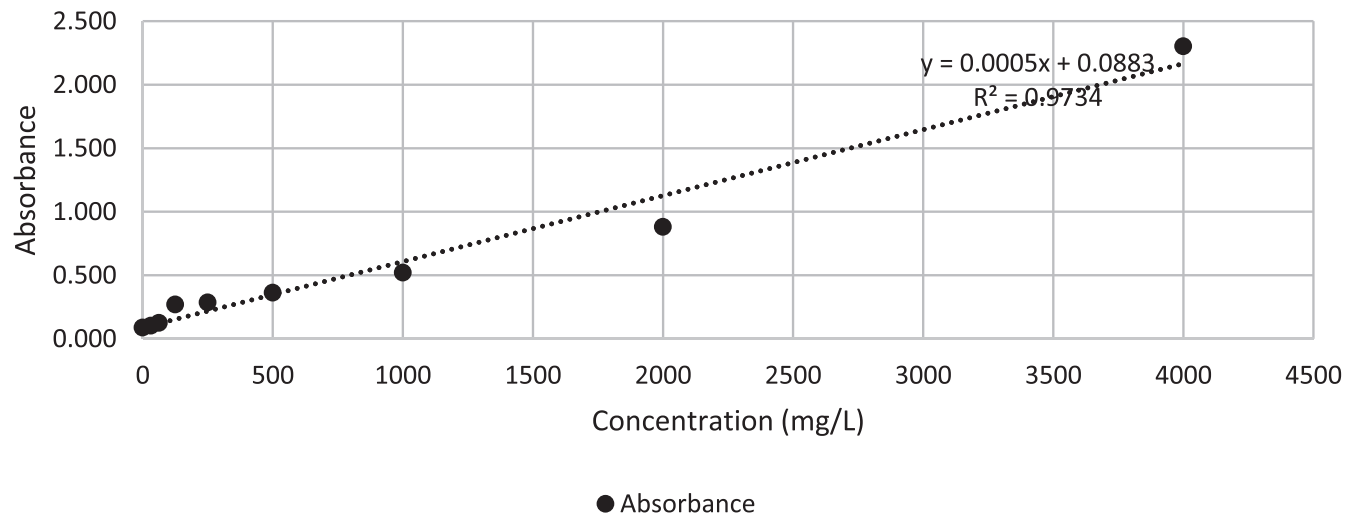




\section{ACKNOWLEDGEMENT}

The authors would like to express their gratitude to the Faculty of Medicine and Health Sciences for the research and from the Department of Nutrition for the technical assistance rendered.

\section{REFERENCES}

Adaramoye, O.A. \& Akanni, O.O. 2016. Effects of long-term administration of aspartame on biochemical indices, lipid profile and redox status of cellular system of male rats. Journal of Basic and Clinical Physiology and Pharmacology, 27(1): 29-37.

Bartoszek, M., Polak, J. \& Chorążewski, M. 2018. Comparison of antioxidant capacities of different types of tea using the spectroscopy methods and semi-empirical mathematical model. European Food Research and Technology, 244(4): 595-601.

Benzie, I.F.F. \& Strain, J.J. 1996. The Ferric Reducing Ability of Plasma (FRAP) as a Measure of "Antioxidant Power": The FRAP Assay. Analytical Biochemistry, 239(1): 70-76.

Bhagat, A.R., Delgado, A.M., Issaoui, M., Chammem, N., Fiorino, M., Pellerito, A. \& Natalello, S. 2019. Review of the role of fluid dairy in delivery of polyphenolic compounds in the diet: Chocolate milk, coffee beverages, matcha green tea, and beyond. Journal of AOAC International, 102(5), 1365-1372.

Chattopadhyay, S., Raychaudhuri, U. \& Chakraborty, R. 2014. Artificial sweeteners - A review. Journal of Food Science and Technology, 51(4): 611-621.

Chu, C., Deng, J., Man, Y. \& Qu, Y. 2017. Green Tea Extracts Epigallocatechin-3-gallate for Different Treatments. BioMed Research International, 2017: 5615647.

Chu, K.O. \& Pang, C.C.P. 2018. Pharmacokinetics and Disposition of Green Tea Catechins. IntechOpen.

Cutrim, C.S. \& Cortez, M.A.S. 2018. A review on polyphenols: Classification, beneficial effects and their application in dairy products. International Journal of Dairy Technology, 71(3): 564-578.

Egert, S., Tereszczuk, J., Wein, S., Müller, M.J., Frank, J., Rimbach, G. \& Wolffram, S. 2013. Simultaneous ingestion of dietary proteins reduces the bioavailability of galloylated catechins from green tea in humans. European Journal of Nutrition, 52(1): 281-288.
Everette, J.D., Bryant, Q.M., Green, A.M., Abbey, Y.A., Wangila, G.W. \& Walker, R.B. 2010. Thorough Study of Reactivity of Various Compound Classes toward the Folin"Ciocalteu Reagent. Journal of Agricultural and Food Chemistry, 58(14), 8139-8144.

Hayat, K., Iqbal, H., Malik, U., Bilal, U. \& Mushtaq, S. 2015. Tea and its consumption: Benefits and risks. Critical Reviews in Food Science and Nutrition, 55(7): 939-954.

Hertog, M.G.L., Sweetnam, P.M., Fehily, A.M., Elwood, P.C. \& Kromhout, D. 1997. Antioxidant flavonols and ischemic heart disease in a Welsh population of men: The caerphilly study. American Journal of Clinical Nutrition, 65(5): 1489-1494.

Hollman, P.C.H., Hof, K.H.V.H., Tijburg, L.B.M. \& Katan, M.B. 2001. Addition of milk does not affect the absorption of flavonols from tea in man. Free Radical Research, 34(3): 297-300.

Horie, H., Ema, K. \& Sumikawa, O. 2017. Chemical components of matcha and powdered green tea. Journal of Cookery Science of Japan, 50(5): 182-188.

Imran, A., Sadiq Butt, M., Saeed, F., Sajid Arshad, M., Sultan, T. \& Sohaib, M. 2017. Effect of different time-solvent interactions on polyphenol content of milky tea. Journal of Food Processing and Preservation, 41(4): 3-6.

Kartsova, L.A. \& Alekseeva, A.V. 2008. Effect of milk caseins on the concentration of polyphenolic compounds in tea. Journal of Analytical Chemistry, 63(11): 1107-1111.

Koláčková, T., Kolofiková, K., Sytačová, I., Snopek, L., Sumczynski, D. \& Orsavová, J. 2020. Matcha tea: Analysis of nutritional composition, phenolics and antioxidant activity. Plant Foods for Human Nutrition, 75(1): 48-53.

Korir, M.W., Wachira, F.N., Wanyoko, J.K., Ngure, R.M. \& Khalid, R. 2014. The fortification of tea with sweeteners and milk and its effect on in vitro antioxidant potential of tea product and glutathione levels in an animal model. Food Chemistry, 145: 145-153.

Ku, K.M.O., Choi, J.N., Kim, J., Kee Kim, J., Yoo, L.G., Lee, S.J., Hong, Y.S. \& Lee, C.H. 2010. Metabolomics analysis reveals the compositional differences of shade grown tea (Camellia sinensis L.). Journal of Agricultural and Food Chemistry, 58(1): 418-426.

Kumarihami, H.M.P.C. \& Song, K.J. 2018. Review on challenges and opportunities in global tea industry. The Korean Tea Society, 24(3): 79-87. 
Kyle, J.A.M., Morrice, P.C., McNeill, G. \& Duthie, G.G. 2007. Effects of infusion time and addition of milk on content and absorption of polyphenols from black tea. Journal of Agricultural and Food Chemistry, 55(12): 4889-4894.

Langley-Evans, S.C. 2000. Consumption of black tea elicits an increase in plasma antioxidant potential in humans. International Journal of Food Sciences and Nutrition, 51(5): 309-315.

Lee, L.S., Kim, S.H., Kim, Y.-B. \& Kim, Y.-C. 2014. Quantitative analysis of major constituents in green tea with different plucking periods and their antioxidant activity. Molecules, 19(7): 91739186.

Leenen, R., Roodenburg, A.J.C., Tijburg, L.B.M. \& Wiseman, S.A. 2000. A single dose of tea with or without milk increases plasma antioxidant activity in humans. European Journal of Clinical Nutrition, 54(1): 87-92.

Li, X. \& Zhu, X. 2015. Tea: Types, production, and trade. In Encyclopedia of Food and Health. B. Caballero, P.M. Finglas and F. Toldrá (Eds.). Academic Press. pp. 279-282.

Lorenz, M., Jochmann, N., Von Krosigk, A., Martus, P., Baumann, G., Stangl, K. \& Stangl, V. 2007. Addition of milk prevents vascular protective effects of tea. European Heart Journal, 28(2): 219-223.

Mirzaei, A. \& Mirzaei, M. 2013. The effects of adding milk on antioxidant activities of green and black tea. Bulletin of Environment, Pharmacology and Life Sciences, 2(5): 37-42.

Musial, C., Kuban-Jankowska, A. \& GorskaPonikowska, M. 2020. Beneficial properties of green tea catechins. International Journal of Molecular Sciences, 21(5): 1744.

Muthuiah, M.J., Thomas, J., Kumar, R.R. \& Azad Mandal, A.K. 2009. Studies on radical scavenging activity of tea leaves and effect of additives on activities of black tea liquor. International Journal of Food Science and Technology, 44(10): 2070-2074.

Otemuyiwa, I.O., Williams, M.F. \& Adewusi, S.A. 2017. Antioxidant activity of health tea infusions and effect of sugar and milk on in vitro availability of phenolics in tea, coffee and cocoa drinks. Nutrition and Food Science, 47(4): 458-468.

Prasanth, M.I., Sivamaruthi, B.S., Chaiyasut, C. \& Tencomnao, T. 2019. A review of the role of green tea (Camellia sinensis) in antiphotoaging, stress resistance, neuroprotection, and autophagy. Nutrients, 11(2): 474.
Ramírez-Aristizabal, L.S., Ortíz, A., RestrepoAristizabal, M.F. \& Salinas-Villada, J.F. 2017. Comparative study of the antioxidant capacity in green tea by extraction at different temperatures of four brands sold in Colombia. Vitae, 24(2): 132-145.

Rashidinejad, A., Birch, E.J. \& Everett, D.W. 2016 a. Interactions between milk fat globules and green tea catechins. Food Chemistry, 199: 347-355.

Rashidinejad, A., Birch, E.J. \& Everett, D.W. 2016 b. The behaviour of green tea catechins in a fullfat milk system under conditions mimicking the cheesemaking process. International Journal of Food Sciences and Nutrition, 67(6): 624-631.

Reddy, V.C., Sagar, G.V.V. Sreeramulu, D., Venu, L. \& Raghunath, M. 2005. Addition of milk does not alter the antioxidant activity of black tea. Annals of Nutrition and Metabolism, 49(3): 189-195.

Reygaert, W.C. 2018. Green tea catechins: Their use in treating and preventing infectious diseases. BioMed Research International, 2018: 9105261.

Richelle, M., Tavazzi, I. \& Offord, E. 2001. Comparison of the antioxidant activity of commonly consumed polyphenolic beverages (coffee, cocoa, and tea) prepared per cup serving. Journal of Agricultural and Food Chemistry, 49(7): 3438-3442.

Ryan, L. \& Petit, S. 2010. Addition of whole, semiskimmed, and skimmed bovine milk reduces the total antioxidant capacity of black tea. Nutrition Research, 30(1): 14-20.

Ryan, L. \& Sutherland, S. 2011. Comparison of the effects of different types of soya milk on the total antioxidant capacity of black tea infusions. Food Research International, 44(9): 3115-3117.

Sakurai, K., Shen, C., Ezaki, Y., Inamura, N., Fukushima, Y., Masuoka, N. \& Hisatsune, T. 2020. Effects of matcha green tea powder on cognitive functions of community-dwelling elderly individuals. Nutrients, 12(12): 3639.

Shalaby, E.A., Mahmoud, G.I. \& Shanab, S.M.M. 2016. Suggested mechanism for the effect of sweeteners on radical scavenging activity of phenolic compounds in black and green tea. Frontiers in Life Science, 9(4): 241-251.

Sharma, V., Vijay Kumar, H. \& Jagan Mohan Rao, L. 2008. Influence of milk and sugar on antioxidant potential of black tea. Food Research International, 41(2): 124-129.

Siebert, K.J., Troukhanova, N.V. \& Lynn, P.Y. 1996. Nature of polyphenol - protein interactions. Journal of Agricultural and Food Chemistry, 44(1): 80-85. 
Singleton, V.L. \& Rossi, J.A. 1965. Colorimetry of total phenolics with phosphomolybdicphosphotungstic acid reagents. American Journal of Enology and Viticulture, 16: 144-158.

Tewari, S., Gupta, V. \& Bhattacharya, S. 2000. Comparative study of antioxidant potential of tea with and without additives. Indian Journal of Physiology and Pharmacology, 44(2): 215-219.

Van Het Hof, K.H., Kivits, G.A.A., Weststrate, J.A. \& Tijburg, L.B.M. 1998. Bioavailability of catechins from tea: The effect of milk. European Journal of Clinical Nutrition, 52(5): 356-359.

Wipatanawin, A., Phongsawanit, S., Maneeratprasert, T., Lertsiri, S. \& Deetae, P. 2015. Determination of the effects of adding milk and sugar on the antioxidant capacity of oolong tea by chemical and cell culture-based antioxidant assays. Chiang Mai Journal of Science, 42(3): 699-711.
Xiao, F., Xu, T., Lu, B. \& Liu, R. 2020. Guidelines for antioxidant assays for food components. Food Frontiers, 1(1): 60-69.

Xie, Y., Kosiñska, A., Xu, H. \& Andlauer, W. 2013. Milk enhances intestinal absorption of green tea catechins in in vitro digestion/Caco-2 cells model. Food Research International, 53(2): 793800.

Yan, Z., Zhong, Y., Duan, Y., Chen, Q. \& Li, F. 2020. Antioxidant mechanism of tea polyphenols and its impact on health benefits. Animal Nutrition, 6(2): 115-123.

Ye, J., Fan, F., Xu, X. \& Liang, Y. 2013. Interactions of black and green tea polyphenols with whole milk. Food Research International, 53(1): 449455 . 